\title{
Case control study of risk factors and ophthalmological outcomes of very low birth weight infants with Type 1 retinopathy of prematurity
}

Tryphena Rachel $\mathrm{Ng}^{1}$, MBBS, Inez B Wong ${ }^{2}$, FRCSEd, FRCophth, Cheryl S $\underline{N g o}^{2}$, MMed, FRCSEd, Krishnamoorthy Niduvaje ${ }^{3,4}$, MRCP, FAMS, Xin Ying $\underline{\text { Ngiam }}^{3}$, MMed, MRCPCH, Sonoko Sensaki ${ }^{3}$, MD, MPH, Le Ye $\underline{\underline{L e}} \underline{e}^{3,4}, \mathrm{MM} e d, \mathrm{MRCPCH}$

INTRODUCTION We evaluated the risk factors associated with Type 1 retinopathy of prematurity (ROP) in very low birth weight (VLBW) infants and compared ophthalmologic outcomes between cases with Type 1 ROP who received treatment and gestational age-matched controls with mild or no ROP not requiring treatment.

METHODS This was a retrospective case-control study of VLBW infants born in National University Hospital, Singapore, from January 2001 to December 2013. 17 cases with Type 1 ROP were each matched for gestational age with controls who had either mild (below Stage 2) or no ROP. Antenatal, perinatal and postnatal variables, as well as childhood ophthalmologic outcomes, were collected from their clinical records and analysed.

RESULTS The number of packed cell transfusions and highest fraction of inspired oxygen given at weeks 7-10 were found to be statistically significant on multivariate analysis ( $p=0.045$ and $p=0.049$, respectively). None of the infants had blindness or retinal detachment, and there were no significant differences in refractive errors between the groups at 1-4 years of age. Strabismus at four years of age was more common in the group with Type 1 ROP $(p=0.023)$.

CONCLUSION: Increased episodes of blood transfusions and chronic lung disease requiring high oxygen supplementation at 7-10 weeks of life are significant risk factors associated with Type 1 ROP in VLBW infants in our study. Strabismus at four years is more common in this group of patients. This study highlights the importance of long-term ophthalmologic surveillance for these high-risk children.

Keywords: chronic lung disease, strabismus, transfusion

\section{INTRODUCTION}

Retinopathy of prematurity (ROP) is a leading cause of blindness in premature infants, especially affecting the smallest and most premature ones. As technology gets more advanced and the level of care improves across neonatal units globally, more very preterm infants will survive, which in turn may lead to increased cases of ROP. Although ROP affects infants who are most premature and of the lowest birth weights, only a fraction of these very preterm infants develop ROP (36.9\% of infants in the Early Treatment for Retinopathy of Prematurity [ETROP] study ${ }^{(1)}$ and $27.1 \%$ of infants in the Cryotherapy for Retinopathy of Prematurity [CRYO-ROP] study ${ }^{(2)}$ developed severe ROP [sROP], as defined in the Methods section of this paper).

ROP develops when there is early hyperoxic damage to the retina and subsequent uncontrolled neovascularisation of the immature retinal vasculature. ${ }^{(3,4)}$ It is often found in infants with low birth weight, small gestational age (GA) and prolonged exposure to supplementary oxygen. ${ }^{(5)}$ While hyperoxygenation is a known risk factor, safe limits of oxygenation have not been clearly defined ${ }^{(6)}$ and remain controversial. ${ }^{(7,8)}$ Aside from the degree of prematurity and oxygen saturations, centres around the world have found various risk factors contributing to the development of ROP, including sepsis, invasive ventilation, presence of respiratory distress syndrome, patent ductus arteriosus, poor postnatal weight gain and the number of blood transfusions. However, the findings remain inconsistent across

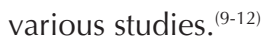

Our study thus sought to elucidate the risk factors that are most important in the development of ROP in very low birth weight (VLBW) infants in our multicultural population so that these can be mitigated to reduce the rates of ROP in our population. Additionally, as all infants in our study with Type 1 ROP were treated with laser therapy, we aimed to determine ophthalmologic outcomes in early childhood for treated ROP in our population to assist in prognostication and comparison of ophthalmologic outcomes between infants with and without treated ROP.

\section{METHODS}

This was a retrospective, matched case-control study that aimed to investigate sROP in VLBW infants. Inclusion criteria for both cases and controls were VLBW (i.e. $\leq 1,500 \mathrm{~g}$ ) infants with GA < 32 weeks born in National University Hospital, Singapore, from January 2001 to December 2013, who survived till their first ophthalmological examination at six weeks or $>36$ weeks, as per the Royal College of Ophthalmologists (RCO) guidelines. The study was approved by the National Healthcare Group

\footnotetext{
${ }^{1}$ Khoo Teck Puat-National University Children's Medical Institute, ${ }^{2}$ Department of Ophthalmology, ${ }^{3}$ Department of Neonatology, National University Hospital, National University Health System, ${ }^{4}$ Department of Paediatrics, Yong Loo Lin School of Medicine, National University of Singapore, Singapore

Correspondence: Dr Lee Le Ye, Senior Consultant, Department of Neonatology, National University Hospital, 1 E Kent Ridge Road, NUHS Tower Block, Singapore 119228. le_ye_lee@nuhs.edu.sg
} 
Domain Specific Review Board (NHG DSRB Ref: 2014/00372). All of these infants in our centre received routine screening for ROP via indirect binocular ophthalmoscopy by trained paediatric ophthalmologists, as per RCO guidelines. ${ }^{(10)}$ Details of the screening schedule are shown in Box $1 .^{(13)}$ Retinopathy was graded according to the International Classification of ROP(11) $\left(B o x 2^{(14)}\right)$. The risk factors were studied retrospectively.

Infants who had sROP were chosen as cases. SROP was defined by: (a) threshold disease as defined by the CRYO-ROP study, ${ }^{(2)}$ used by our centre before 2008, i.e. five contiguous or eight total clock hours of Stage 3 in Zone I or Zone II with plus disease; or (b) Type I ROP as per the ETROP(1) study, implemented in our centre in 2008 till the present, i.e. Zone I, any stage with plus; Zone I, Stage 3 without plus; and Zone II, Stage 2-3 with plus. These infants were deemed to have sROP that would not regress spontaneously and, hence, needed treatment. A total of 17 infants who met these criteria within the study period were identified as cases. All 17 cases received laser retinal photocoagulation; four received laser therapy to only one eye, while the other 13 received therapy to both eyes.

As extreme early GA and extremely low birth weight are two of the major factors that are associated with sROP, we adopted a GA-matched case-control design to determine which other perinatal factors besides these two influenced the development of sROP. The cases were each matched to two controls of similar $\mathrm{GA}$, giving a total of 34 controls. Controls were infants who met the inclusion criteria stated above but did not have sROP, i.e. they had no or mild ROP that did not require treatment. Cases were matched to controls that were born within two years of each other to minimise differences in management. When controls of the exact GA could not be found, infants younger or older by one week of the GA were chosen.

For the first part of the study, data on antenatal, perinatal and postnatal factors was collected by reviewing their case records and charts. Vital parameters such as pulse oximetry reading $\left(\mathrm{SpO}_{2}\right)$ were collated from nursing charts, which included hourly pointof-care vital recordings. Fraction of inspired oxygen $\left(\mathrm{FiO}_{2}\right)$ and ventilation data was further subcategorised into different time periods (weeks 1-2, weeks 3-6, weeks 7-10 and beyond week 10 of life), as development of ROP is closely linked to hypoxia and hyperoxia at different phases of life.

Data was analysed using IBM SPSS Statistics version 22.0 (IBM Corp, Armonk, NY, USA) to determine the factors that statistically increased the risk of developing sROP. Both univariate and multivariate analyses were performed. For the univariate analysis, two-tailed Fisher's exact test and odds ratio calculations were used for nominal variables, the MannWhitney $U$ test for ordinal/non parametric variables, and the independent $t$-test for parametric variables. Binary logistic regression was used for multivariate analysis. A p-value $<0.05$ was taken as statistically significant.

For the second part, we aimed to study ophthalmologic outcomes. All surviving VLBW infants were given follow-up appointments with a paediatrician and an ophthalmologist. We reviewed the clinical records of their follow-up visits to
Box 1. Screening schedule for retinopathy of prematurity (ROP) for all infants $\leq 1,500 \mathrm{~g}$ with a gestational age of $<32$ weeks, according to the guidelines of the Royal College of Ophthalmologists: ${ }^{(13)}$

- All neonates $<32$ weeks' gestational age (up to 31 weeks and six days) or less than 1,501 $\mathrm{g}$ birth weight were screened for ROP

- Neonates born before 27 weeks' gestational age - the first ROP screening examination is done at 30-31 weeks' postmenstrual age - Neonates born between 27 and 32 weeks' gestational age - the first ROP screening examination is done at $4-5$ weeks' postnatal age

-Weekly screenings when:

- the vessels end in Zone I or posterior Zone II;

- there is any plus or pre-plus disease;

- there is any Stage 3 disease in any zone

- Two-weekly screenings for all other circumstances, until the following criteria for termination is reached and the infant is no longer at risk for sight-threatening ROP:

- In infants without ROP, screening can stop when vascularisation has extended into Zone III, usually after 36 completed weeks' postmenstrual age

- In infants with ROP, screening for progressive active disease can stop when there are signs of regression on at least two successive examinations:

o Lack of increase in severity. o Partial resolution progressing towards complete resolution. o Change in colour in the ridge from salmon pink to white o Transgression of vessels through the demarcation line o Commencement of the process of replacement of active ROP lesions by scar tissue

\section{Box 2. International classification of retinopathy of} prematurity (ROP): ${ }^{(14)}$

ROP described in terms of:

-Zone

- Zone I: circle from the centre of disc with radius of twice the distance from disc to macula

- Zone II: from nasal edge of Zone I to ora nasally and up to equator region of retina temporally

- Zone III: from temporal crescent of retina anterior to Zone II - Stage

- Stage 1: demarcation line

- Stage 2: ridge with height and width

-Stage 3: extraretinal fibrovascular proliferation

- Stage 4A: extrafoveal retinal detachment

- Stage 4B: subtotal retinal detachment involving the fovea

- Stage 5: total retinal detachment

- Presence of plus disease: marked engorgement of posterior veins and tortuosity of arterioles

- Presence of pre-plus disease: vascular abnormalities of the posterior pole that are insufficient for the diagnosis of plus disease, but that demonstrate more arterial tortuosity and more venous dilatation than normal

the attending ophthalmologist and collected data on their ophthalmologic outcomes (Box 3).

As Southeast Asian children tend to have higher rates of myopia compared to their Western counterparts, we chose to use the definitions of refractive error from a local study, the Strabismus, Amblyopia, and Refractive Error in Young Singaporean Children (STARS) study, ${ }^{(14)}$ a large-scale study involving 3,009 Singaporean 
Box 3. Ophthalmological outcomes (from 1 to 4 years of age):

- Blindness - worse than 6/60 vision, as per legal definitions

- Structural outcomes - any retinal detachment or other abnormalities

- Ocular alignment - any manifest strabismus, i.e. tropias as defined by constant or intermittent heterotropia of any magnitude at any distance; tested by using the unilateral cover (cover-uncover) test, alternate cover and prism tests

- Refractive errors as determined by cycloplegic refraction using definitions in the Strabismus, Amblyopia and Refractive Error in Young Singaporean Children study ${ }^{(14)}$

- Presence of myopia (any eye having spherical equivalence [SE] $\leq-0.50 \mathrm{D}$ ), high myopia (SE $\leq-6.00 \mathrm{D}$ )

- Presence of hyperopia (any eye having $\mathrm{SE} \geq+3.00 \mathrm{D}$ )

- Presence of astigmatism (any eye having cylinder $\geq+1.50 \mathrm{D}$ )

- Anisometropia (SE difference between each eye $\geq 2.00 \mathrm{D}$ ) were calculated

children aged 6-72 months who were examined from 2006 to 2008. Data on the above parameters was retrospectively extracted from the records of clinic follow-up visits done at 12-18 months chronological age and at approximately two years, three years and four years of age.

Four of the cases received laser treatment to one eye, while the rest of the cases received laser treatment to both eyes. Regardless of the number of eyes treated, the outcome of the 'worst eye' of each case was analysed (e.g. the eye with the higher refractive error or structural outcome) and compared against the 'worst eye' of the controls. Two-tailed Fisher's exact test and odds ratio calculations were used to determine if infants with sROP, albeit treated, showed any differences in short-term ophthalmologic outcomes as compared to their peers without sROP. Cases that received laser treatment to one eye were also separately analysed to determine if there were any differences in refractive errors between the eyes or in the development of strabismus or amblyopia.

\section{RESULTS}

Our unit is a 20-bedded neonatal intensive care unit with type C intensive care. A total of $597 \mathrm{VLBW}$ infants were admitted from 2001 to 2013, with an average survival rate of $92 \%$ and a yearly sROP incidence of $5.1 \%-8.3 \%$. Among these VLBW infants, the mean gestation period was $28.8 \pm 2.0$ weeks and the mean birth weight was $1,106.08 \mathrm{~g} \pm 236.11 \mathrm{~g}$. About $35 \%$ of these were extremely low birth weight $(<1,000 \mathrm{~g})$ infants.

The mean GA of the 17 cases and 34 controls was $25.7 \pm 1.5$ weeks and $26.0 \pm 1.2$ weeks, respectively, indicating no significant differences between them $(p=0.36)$. Mean birth weights between the cases and controls were also comparable (812 g \pm 167 g vs. $865 \mathrm{~g} \pm 167 \mathrm{~g} ; \mathrm{p}=0.27$ ). Thus, both groups were appropriately matched for GA and birth weight. Our subjects were predominantly Asian with the following race profiles: cases $-64.7 \%$ Chinese, $17.6 \%$ Malay, $5.9 \%$ Indian and $5.9 \%$ Bangladeshi; controls - 52.9\% Chinese, $17.6 \%$ Malay, $23.5 \%$ Indian, 2.9\% Korean and 2.9\% Caucasian.

Table I shows the antenatal, perinatal and postnatal risk factors in our study population. Antenatal factors were not shown to have significant association with the development of sROP. Among the perinatal factors, Apgar score at five minutes showed statistical significance $(p=0.007)$, with a median Apgar score at five minutes of 8 for controls and 9 for cases; however, this was unlikely to be of clinical significance. Several postnatal risk factors differed significantly between the cases and controls. In our population, infection-related factors showed a strong positive association with the development of sROP. These were cultureproven sepsis $(35.3 \%$ of cases vs. $8.8 \%$ of controls; $p=0.045$, odds ratio [OR] 5.63, 95\% confidence interval [CI] 1.20-26.48) and necrotising enterocolitis Stage Ilb and above (23.5\% of cases vs. $2.9 \%$ of controls; $p=0.037$, OR 10.51, 95\% Cl 1.03-99.60). Gastrointestinal perforation that was isolated also showed a strong positive association $(23.5 \%$ of cases vs. $0 \%$ of controls; $p=0.010$, OR 23.0, 95\% Cl 1.21-456.82), suggesting that the more severe the necrotising enterocolitis, the higher the odds of sROP (Table I). Furthermore, cases with SROP had a higher median number of red blood cell transfusions than the controls, at 4 vs. 2, and this was statistically significant $(p<0.001)$.

Among the respiratory factors studied, early ventilation and oxygenation settings showed no significant differences between the two groups. However, cases with sROP had more prolonged days of invasive ventilation and higher fractional oxygenation requirements in the later phases. Cases were intubated for significantly longer periods of time (median 20 days for cases vs. 5 days for controls, $p=0.002$ ) and on oxygen therapy for significantly longer periods (median 35.5 days for cases vs. 6.0 days for controls, $p=0.006$; Table I). Medians were used for these variables instead of means, as there were outliers to the data, with two cases being intubated for more than three months (whereas most of the other cases were only intubated for a few weeks) and one case on oxygen therapy for more than a year.

Both cases and controls had similar oxygen requirements in the first two weeks ( $41 \%$ for cases vs. $49 \%$ for controls, $p=0.866$ ). Beyond the first two weeks of life, cases with sROP also had higher oxygen requirements, requiring medians of 35\%, 30\% and $25 \%$ oxygen at 3-6 weeks, 7-10 weeks and beyond 10 weeks, respectively. From the third week of life onwards, the median $\mathrm{FiO}_{2}$ given to controls was $21 \%$, i.e. the majority of the infants did not require supplemental oxygen.

Cases demonstrated prolonged respiratory instability, with $76.5 \%$ of them having chronic lung disease with the need for oxygen or respiratory support at 36 weeks compared to $44.1 \%$ of controls ( $p=0.039$, OR 4.11, 95\% Cl 1.11-15.24). Significantly, more cases continued to have episodes of apnoea and bradycardia at the postmenstrual age (PMA) of 36 weeks and beyond $(76.5 \%$ of cases vs. $20.5 \%$ of controls; $p<0.001$, OR $12.5,95 \% \mathrm{Cl} 3.10-50.62)$. The presence of respiratory distress syndrome, pulmonary haemorrhage and pneumothorax did not show any significant effects on the severity of ROP. None of the neurological or cardiac risk factors studied showed significant differences between the cases and controls in our population.

We further analysed the growth of the cases and controls on discharge and found that cases were discharged after longer hospital stays, at an average PMA of 43.9 weeks compared to 38.4 
Table I. Antenatal, perinatal and postnatal risk factors of the study cohort.

\begin{tabular}{|c|c|c|c|c|c|}
\hline \multirow[t]{2}{*}{ Parameter } & \multicolumn{2}{|c|}{$\% /$ mean \pm SD } & \multirow[t]{2}{*}{ OR $(95 \% \mathrm{Cl})$} & \multirow[t]{2}{*}{ p-value* } & \multirow[t]{2}{*}{ p-value ${ }^{\dagger}$} \\
\hline & Cases $(n=17)$ & Controls $(n=34)$ & & & \\
\hline \multicolumn{6}{|l|}{ Antenatal } \\
\hline Gender & & & NIL & 0.254 & \\
\hline Male & 58.8 & 41.2 & & & \\
\hline Female & 41.2 & 58.8 & & & \\
\hline Type of delivery & & & NIL & 0.232 & \\
\hline Normal vaginal delivery & 70.6 & 50.0 & & & \\
\hline Lower segment Caesarean section & 29.4 & 50.0 & & & \\
\hline Pre-labour prolonged rupture of membranes & 52.9 & 52.9 & $1.00(0.31-3.21)$ & 1.000 & \\
\hline Antepartum haemorrhage/abruptio placentae & 41.2 & 29.4 & $1.68(0.49-5.66)$ & 0.531 & \\
\hline Fetal distress & 29.4 & 8.8 & $4.30(0.88-20.88)$ & 0.099 & \\
\hline Pregnancy-induced hypertension/preeclampsia & 5.9 & 14.7 & $0.36(0.03-3.37)$ & 0.650 & \\
\hline Maternal pyrexia & 23.5 & 5.9 & $4.90(0.80-30.20)$ & 0.087 & \\
\hline Antenatal steroids completed $>24 \mathrm{hr}$ before birth & $66.7(n=15)$ & $66.7(n=33)$ & $1.00(0.29-3.39)$ & 1.000 & \\
\hline \multicolumn{6}{|l|}{ Perinatal } \\
\hline Gestational (postmenstrual) age (wk) & $25.7 \pm 1.5$ & $26.1 \pm 1.2$ & NIL & 0.360 & \\
\hline Birth weight (g) & $812 \pm 167$ & $865 \pm 167$ & NIL & 0.270 & \\
\hline Weight z-score at birth & $0.15 \pm 0.87$ & $0.24 \pm 0.70$ & NIL & 0.710 & \\
\hline Median Apgar score at $1 \mathrm{~min}$ & 5 & 6.5 & NIL & 0.488 & \\
\hline Median Apgar score at $5 \mathrm{~min}$ & 8 & 9 & NIL & 0.007 & $\begin{array}{l}\text { Not } \\
\text { analysed }\end{array}$ \\
\hline Intubation at birth & 76.5 & 88.2 & $0.43(0.09-2.00)$ & 0.410 & \\
\hline Mean neonatal temperature at birth $\left({ }^{\circ} \mathrm{C}\right)$ & $33.0(n=12)$ & $35.9(n=28)$ & NIL & 0.864 & \\
\hline Hypothermic $\left(<36^{\circ} \mathrm{C}\right)$ at birth & $58.3(n=12)$ & $60.7(n=28)$ & $0.96(0.32-2.91)$ & 1.000 & \\
\hline Clinical Risk Index for Babies II score & $12.5 \pm 2.3(n=12)$ & $11.1 \pm 2.4(n=28)$ & NIL & 0.102 & \\
\hline $\mathrm{pH}$ at birth & $7.27 \pm 0.12$ & $7.27 \pm 0.12$ & NIL & 0.919 & \\
\hline Base excess at birth & $-5.4 \pm 5.07$ & $-6.29 \pm 4.94$ & NIL & 0.535 & \\
\hline \multicolumn{6}{|l|}{ Postnatal } \\
\hline \multicolumn{6}{|l|}{ Neurological } \\
\hline $\begin{array}{l}\text { Median worse grade of intraventricular } \\
\text { haemorrhage (worst grade on any side) }\end{array}$ & 0 & 1 & NIL & 0.870 & \\
\hline $\begin{array}{l}\text { Severe intraventricular haemorrhage (Grade III or } \\
\text { more on any side) }\end{array}$ & 17.6 & 5.9 & $3.42(0.51-22.80)$ & 0.318 & \\
\hline Periventricular leukomalacia & 17.6 & 2.9 & $7.07(0.67-73.90)$ & 0.102 & \\
\hline \multicolumn{6}{|l|}{ Infections } \\
\hline Sepsis (proven with positive blood cultures) & 35.3 & 8.8 & $5.63(1.20-26.48)$ & 0.045 & 0.843 \\
\hline Necrotising enterocolitis (Stage IIB and above) & 23.5 & 2.9 & $10.51(1.03-99.60)$ & 0.037 & 1.000 \\
\hline Gastrointestinal perforation isolated & 23.5 & 0.0 & $23.0(1.21-456.82)$ & 0.010 & 0.998 \\
\hline \multicolumn{6}{|l|}{ Haematological } \\
\hline Median no. of red blood cell transfusions given & 4 & 2 & NIL & $<0.001$ & 0.045 \\
\hline \multicolumn{6}{|l|}{ Cardiovascular } \\
\hline Significant hypotension requiring inotropes & 23.5 & 9.1 & $3.07(0.60-15.70)$ & 0.210 & \\
\hline Patent ductus arteriosus & 94.1 & 73.5 & $5.76(0.66-49.90)$ & 0.135 & \\
\hline Cardiac anomalies e.g. ventricular septal defect & 11.8 & 17.6 & $0.62(0.11-3.47)$ & 0.703 & \\
\hline \multicolumn{6}{|l|}{ Respiratory } \\
\hline Respiratory distress syndrome & 82.4 & 88.2 & $0.62(0.12-3.16)$ & 0.673 & \\
\hline Pulmonary haemorrhage & 17.6 & 2.9 & $7.07(0.67-73.90)$ & 0.102 & \\
\hline Pneumothorax & 5.9 & 11.8 & $0.46(0.04-4.55)$ & 0.654 & \\
\hline $\begin{array}{l}\text { Chronic lung disease by } 28 \text { days definition } \\
\text { (requiring supplemental oxygen or mechanical } \\
\text { ventilation at } 28 \text { days of life and beyond) }\end{array}$ & 94.1 & 88.2 & $2.133(0.22-20.72)$ & 0.654 & \\
\hline
\end{tabular}


Table I. (Contd...)

\begin{tabular}{|c|c|c|c|c|c|}
\hline \multirow[t]{2}{*}{ Parameter } & \multicolumn{2}{|c|}{$\% /$ mean \pm SD } & \multirow[t]{2}{*}{ OR $(95 \% \mathrm{Cl})$} & \multirow[t]{2}{*}{ p-value* } & \multirow[t]{2}{*}{ p-value ${ }^{\dagger}$} \\
\hline & Cases $(n=17)$ & Controls $(n=34)$ & & & \\
\hline $\begin{array}{l}\text { Chronic lung disease by } 36 \text { week definition } \\
\text { (requiring supplemental oxygen or mechanical } \\
\text { ventilation at } 36 \text { weeks' postmenstrual age and beyond) }\end{array}$ & 76.5 & 44.1 & $4.11(1.11-15.24)$ & 0.039 & 0.289 \\
\hline $\begin{array}{l}\text { Presence of apnoea and bradycardia at } 36 \text { weeks' } \\
\text { postmenstrual age and beyond }\end{array}$ & 76.5 & 20.5 & $12.5(3.10-50.62)$ & $<0.001$ & 0.624 \\
\hline Median total intubation (day) & 20 & 5 & NIL & 0.002 & 0.162 \\
\hline Median total on oxygen therapy (day) & 35.5 & 6.0 & NIL & 0.006 & 0.407 \\
\hline \multicolumn{6}{|l|}{ Median highest fraction of inspired oxygen required } \\
\hline Week 1-2 & 41 & 49 & NIL & 0.866 & \\
\hline Week 3-6 & 35 & 21 & NIL & 0.035 & 0.130 \\
\hline Week 7-10 & 30 & 21 & NIL & 0.001 & 0.049 \\
\hline Week 10 and beyond & 25 & 21 & NIL & 0.001 & 0.696 \\
\hline \multicolumn{6}{|l|}{ Discharge statistics and postnatal weight gain } \\
\hline Length of hospitalisation (day) & $128.2 \pm 47.0$ & $92.6 \pm 39.5$ & NIL & $<0.001$ & \multirow{3}{*}{$\begin{array}{l}\text { Not } \\
\text { analysed }\end{array}$} \\
\hline Postmenstrual age upon discharge (week) & $43.9 \pm 6.4$ & $38.4 \pm 3.8$ & NIL & $<0.001$ & \\
\hline Weight upon discharge $(g)$ & $2,490 \pm 449$ & $2,210 \pm 468$ & NIL & 0.047 & \\
\hline Weight gain per kg birth weight per day (g) & $17.2 \pm 3.5$ & $17.8 \pm 4.4$ & NIL & 0.606 & \\
\hline Weight z-score upon discharge & $-3.75 \pm 1.82$ & $-2.27 \pm 0.95$ & NIL & $<0.001$ & 0.874 \\
\hline
\end{tabular}

No. of cases and controls are 17 and 34, respectively, unless otherwise stated in parenthesis. p-value $<0.05$ is statistically significant. *B By univariate analysis. $†$ By multivariate analysis (only included if factor is significant in univariate analysis). ‡Not analysed, as Apgar score of 8 vs. 9 was not clinically significant. §Not analysed, as prolonged hospitalisation stays was likely a result of severity of illness rather than a contributing factor to retinopathy of prematurity. Cl: confidence interval; OR: odds ratio; SD: standard deviation

weeks for the controls $(p<0.001)$, with a corresponding average chronological age of 128.2 days vs. 92.6 days $(p<0.001)$. Our analysis of postnatal weight gain found that although the mean weight gain per kg of birth weight per day did not differ significantly between the cases and controls $(17.2 \pm 3.5 \mathrm{~g} / \mathrm{kg} /$ day for cases vs. $17.8 \pm 4.4 \mathrm{~g} / \mathrm{kg} /$ day for controls, $\mathrm{p}=0.606)$, standardised weight z-scores upon discharge differed significantly $(-3.75 \pm 1.82$ for cases vs. $-2.27 \pm 0.95$ for controls, $p<0.001)$. As both groups had weight $z$-scores that were not significantly different at birth $(0.15 \pm 0.87$ for cases vs. $0.24 \pm 0.70$ for controls, $p=0.71)$, the change in weight $z$-scores upon discharge showed a clinically significant postnatal growth failure prior to discharge.

Logistic regression was performed with multivariate analysis using associated factors: culture-proven sepsis; Stage IIB or worse necrotising enterocolitis; gastrointestinal perforation; number of red cell transfusions; weight z-score upon discharge; chronic lung disease at 36 weeks; presence of apnoea and bradycardia at PMA of 36 weeks and beyond; total number of days on oxygen therapy; total number of days intubated; and highest $\mathrm{FiO}_{2}$ given at weeks 3-6, weeks 7-10 and beyond 10 weeks. Apgar score at five minutes, while statistically significant, was not included in the multivariate analysis, as the clinical difference was not clinically relevant. Age and absolute weight upon discharge were also not included in the multivariate analysis, as they were likely a reflection of the subjects' severity of illness, hence requiring longer hospital stays, rather than a factor in the development of sROP. Of these factors, number of red cell transfusions and highest $\mathrm{FiO}_{2}$ given at weeks $7-10$ of life were found to be statistically significant on multivariate analysis $(p=0.049$ and $p=0.045$, respectively). $\mathrm{FiO}_{2}$ given at other weeks of life was not found to be significant on multivariate analysis.

The short-term ophthalmological outcomes up to four years are shown in Table II. Data was available for 15 cases with sROP and 22 controls at $12-18$ months of age; 12 cases and 23 controls at $24 \pm 3$ months; 12 cases and 20 controls at $36 \pm 3$ months; and eight cases and 17 controls at $48 \pm 3$ months. Significant loss-to-follow-up rates were observed in both group: $11 \%$ of cases and $35 \%$ of controls at $12-18$ months; $29 \%$ of cases and $32 \%$ of controls at two years; $29 \%$ of cases and $43 \%$ of controls at three years; and $53 \%$ of cases and $50 \%$ of controls at four years. Of the subjects who were lost to follow up, one control had returned to her home country, while the rest had defaulted the given follow-up appointments.

All of the cases were treated for sROP with only laser therapy without cryotherapy or intravitreal anti-vascular endothelial growth factor (VEGF) injection. Structural and functional outcomes were generally good, both for cases who had ROP and subsequently underwent treatment, as well as for controls who had mild or no ROP without any treatment. None of the subjects were legally blind. Structural outcomes were also good for both groups, with no subjects having retinal detachment.

There were no significant differences in refractive errors between the groups (myopia, hyperopia, astigmatism or anisometropia). None of the subjects had high myopia. There was a significant difference in the presence of manifest strabismus (defined by constant or intermittent heterotropia of any magnitude at any distance) at four years of age, with 50.0\% of cases having any form of tropia and only $5.9 \%$ of controls 
Table II. Retinopathy of prematurity treatment outcomes: refractive errors and strabismus.

\begin{tabular}{|c|c|c|c|c|c|}
\hline \multirow[t]{2}{*}{ Group } & \multicolumn{5}{|c|}{ No. (\%) } \\
\hline & Myopia & Astigmatism & Hyperopia & Anisocoria & Tropia \\
\hline \multicolumn{6}{|l|}{ 12-18 mth } \\
\hline Case $(n=15)^{*}$ & 4/14 (28.6) & $0 / 14(0)$ & $0 / 14(0)$ & $1 / 14(7.1)$ & $3 / 15(20.0)$ \\
\hline Control $(n=22)^{\dagger}$ & $2 / 21(9.5)$ & $2 / 21(9.5)$ & $4 / 21(19.0)$ & $0 / 21(0)$ & $0 / 21(0)$ \\
\hline p-value & 0.191 & 0.506 & 0.133 & 0.400 & 0.059 \\
\hline Odds ratio $(95 \% \mathrm{Cl})$ & $3.80(0.59-24.5)$ & $0.91(0.79-1.04)$ & $0.81(0.66-1.00)$ & $1.08(0.93-1.25)$ & $1.25(0.97-1.61)$ \\
\hline \multicolumn{6}{|l|}{$2 \mathrm{yr} \pm 3 \mathrm{mth}$} \\
\hline Case $(n=12)$ & $5 / 12(41.7)$ & $3 / 12(25.0)$ & $0 / 12(0)$ & 2/12 (16.7) & $3 / 12(25.0)$ \\
\hline Control $(n=23)^{\dagger}$ & $2 / 22(9.1)$ & $3 / 22(13.6)$ & $1 / 22(4.5)$ & $0 / 22(0)$ & $1 / 23(4.3)$ \\
\hline$p$-value & 0.070 & 0.641 & 1.000 & 0.118 & 0.106 \\
\hline Odds ratio $(95 \% \mathrm{Cl})$ & $7.14(1.12-45.5)$ & $2.11(0.35-12.6)$ & $0.95(0.87-1.05)$ & $1.20(0.93-1.55)$ & $7.33(0.67-80.22)$ \\
\hline Case $(n=12)$ & $3 / 12(25.0)$ & $5 / 12(41.7)$ & $0 / 12(0)$ & $1 / 12(8.3)$ & $3 / 12(25.0)$ \\
\hline Control $(n=20)$ & 4/20 (20.0) & $8 / 20(40.0)$ & $0 / 20(0)$ & $0 / 20(0)$ & $1 / 20(5.0)$ \\
\hline$p$-value & 1.000 & 1.000 & NA & 0.375 & 0.136 \\
\hline Odds ratio $(95 \% \mathrm{Cl})$ & $1.33(0.24-7.34)$ & $1.07(0.25-4.59)$ & NA & $1.09(0.92-1.29)$ & $6.33(0.58-69.68)$ \\
\hline \multicolumn{6}{|l|}{$4 \mathrm{yr} \pm 3 \mathrm{mth}$} \\
\hline Case $(\mathrm{n}=8)$ & $2 / 8(25.0)$ & $2 / 8(25.0)$ & $0 / 8(0)$ & $0 / 8(0)$ & $4 / 8(50.0)$ \\
\hline Control $(n=17)^{\ddagger}$ & $3 / 14(21.4)$ & 4/14 (28.6) & $0 / 14(0)$ & $1 / 14(7.1)$ & $1 / 17(5.9)$ \\
\hline p-value & 1.000 & 1.000 & NA & 1.000 & 0.023 \\
\hline Odds ratio $(95 \% \mathrm{Cl})$ & $1.22(0.16-9.47)$ & $0.83(0.12-6.01)$ & NA & $0.93(0.80-1.07)$ & $16.0(1.38-185.4)$ \\
\hline
\end{tabular}

Cycloplegic refraction was not done for: ${ }^{*} 1$ case; +1 control; and $\neq 3$ controls. $p<0.05$ is statistically significant. Cl: confidence interval; $\mathrm{NA}$ : not applicable

having tropias $(\mathrm{p}=0.023$, OR $16.0,95 \% \mathrm{Cl} 1.38-185.4)$. The details of comparison between both groups for refractive errors and strabismus are given in Table II.

Of the four cases who received only laser therapy to one eye instead of both eyes, one case was completely lost to follow-up, and his ophthalmological outcomes are hence unknown. The other three cases all had similar visual outcomes in both eyes despite only one side having received laser treatment. There was no anisometropia, strabismus or gaze preference. Refractive errors were within $0.50 \mathrm{D}$ difference between both eyes throughout all four years of follow-up. Visual acuities in both eyes were similar.

\section{DISCUSSION}

The present study found a number of potential risk factors in preterm extremely low birth weight infants that are consistent with those of studies conducted in other centres. However, interestingly, our data also suggested that weeks $7-10$ of life is a crucial phase in the pathophysiologic development of sROP and that $\mathrm{FiO}_{2}$ given to neonates during this period can affect the development of ROP, with higher $\mathrm{FiO}_{2}$ during this period being significantly associated with sROP.

In our population, late-onset bacterial infections and necrotising enterocolitis showed a strong positive association with development of ROP. This is consistent with findings from various countries such as Hong Kong, ${ }^{(15)} \mathrm{Korea}^{(8)}$ and Canada. ${ }^{(9)}$ This may be due to the production of inflammatory cytokines during infections, causing increased oxidative damage on the immature retina. ${ }^{(16)}$ Onset of sepsis was late (more than three days) for all affected infants (6/17 cases and 3/34 controls), except for one of the cases. This is consistent with a previous study, ${ }^{(17)}$ which showed that late, but not early, neonatal bacteraemia was associated with SROP in extremely-low-GA neonates. While a series of studies done in the United States reported that candida infections have a positive association with ROP, ${ }^{(18,19)}$ this was not demonstrated in our study. Organisms isolated included Enterobacter, coagulase-negative staphylococci and Candida, with no organism showing a particular predominance in this study.

Similar to our findings, studies done in other centres ${ }^{(20,21)}$ have shown an increased number and volume of blood transfusions being significantly associated with the development of ROP. The mechanism of this is controversial. James et al suggested that an increased number of transfusions increases oxygen delivery to the retina due to a lower oxygen affinity of adult haemoglobin in pack cell transfusions, causing more oxidative damage in the immature retina. ${ }^{(22)}$ Cooke et al proposed that transfusions cause secondary iron overload, leading to the increased development of ROP; as transfused cells have a shorter half-life, the iron they store is released as free iron, resulting in the production of more free radicals, which damage the retina. ${ }^{(23)}$

In our study, the prolonged invasive ventilation and oxygenation supplementation that the cases had received as compared to their control counterparts was more likely to be a reflection of the severity of illness than a direct contributor to the development of ROP. This is because many of the cases had chronic lung disease and continued to have prolonged respiratory 
instability with episodes of apnoea or bradycardia even after reaching the GA of 36 weeks.

An interesting finding of the multivariate analysis was that the highest $\mathrm{FiO}_{2}$ given at weeks $7-10$ of life was significantly different between infants who subsequently had sROP and those who did not, whereas $\mathrm{FiO}_{2}$ given at other periods was not significantly different. As the mean GA of our population (both cases and controls) was 26 weeks, at weeks $7-10$ of life, the mean PMA of our subjects was 33-36 weeks. This is the period when subjects are nearing maturity and when one would expect retinal vascularisation to be nearing completion (as the nasal retina becomes vascularised by 36 weeks in normal neonates and temporal retina by about 38-40 weeks), although this process can be delayed in premature neonates. ROP is known to have a two-phase pathogenesis: Phase 1 (after birth to PMA 30 weeks) - vessels growth stops because of hyperoxia; and Phase 2: retinal vascularisation is stimulated by hypoxic retina and oxygen-regulated factors. ${ }^{(24)} \mathrm{A}$ meta-analysis by Chen et al, which summarised previous studies on the relationship between oxygenation and pathogenesis of ROP, suggested that the risk of development of sROP could be lowered with low oxygen saturation $(70 \%-96 \%)$ in the first several postnatal weeks during the first phase of ROP development, whereas high oxygen saturation (94\%-99\%) would be beneficial at $\geq 32$ weeks' PMA, when one would expect the beginning of the second phase of ROP development in which disordered neovascularisation happens as a response to inadequate oxygenation of the retina. ${ }^{(25)}$ However, our study seems to find otherwise. Although theoretically, oxygen in Phase 2 of ROP (PMA 33-36 weeks) could suppress high concentrations of hypoxia-induced oxygen-regulated growth factors, such as VEGF that causes proliferative disease, our study found that supplementing our subjects with higher oxygen concentrations to aim for higher oxygen saturations above $90 \%$ during this period of life may instead be detrimental.

After the tenth week of life, although the majority of cases still needed oxygen supplementation, as compared with the controls who did not, this was not significantly associated with sROP in the multivariate analysis, unlike during weeks $7-10$ of life. This may suggest that weeks $7-10$ of life or PMA 33-36 weeks is possibly a unique period in the development of ROP, and this should be further studied to re-examine what the ideal oxygen saturation target is during this period.

As this is a retrospective study, it is difficult to determine if the increased number of transfusions and the more aggressive ventilation and oxygenation directly contributed to the development of sROP, or if this was because the infants who subsequently developed sROP tended to be sicker and hence needed these interventions.

There is a recent controversy regarding whether targeting a lower oxygen concentration (as advocated to reduce rates of ROP development and other hyperoxic damages) would lead to an overall difference in mortality or morbidity. The 2018 metaanalysis conducted by the Neonatal Oxygenation Prospective Meta-analysis (NeOProM) Collaboration, ${ }^{(26)}$ which analysed five large-scale international randomised double-blind multicentre trials (including a total of 4,965 infants with median GA of 26 weeks and mean birth weight of $832 \mathrm{~g}$ ), concluded that there was no significant difference between targeting lower (85\%-98\%) and higher (91\%-95\%) $\mathrm{SpO}_{2}$ range on the composite primary outcome of death or major disability at a corrected age of 18-24 months. The lower $\mathrm{SpO}_{2}$ target range was associated with a lower risk of sROP requiring treatment but was associated with higher risks of necrotising enterocolitis and death. ${ }^{(26)}$ In light of this controversy, our finding that a PMA of 33-36 weeks would result in less sROP when supplemental oxygen was not given may have implications for target oxygen saturations at different postnatal periods for premature infants.

In addition, our finding of significant postnatal growth failure is in agreement with some recent studies that have also found postnatal weight gain to be an important predictive factor in the development of ROP. Recent studies such as the WINROP, (27) CHOP ROP ${ }^{(28)}$ and ROPScore ${ }^{(29)}$ have come up with algorithms to predict the development of ROP based on postnatal weight gain. The correlation between postnatal weight gain and the development of ROP is believed to be due to postnatal weight gain being a surrogate measure of levels of insulin-like growth factor 1 (IGF-1), with the lack of IGF-1 (and hence low postnatal weight gain) having a positive association with the development of ROP. ${ }^{(30)}$

One group of risk factors that we did not study is genetic risk factors. Holmström et al, in a 2007 meta-analysis of clinical and experimental animal studies, ${ }^{(31)}$ suggested that factors such as sex, presence of genetic polymorphisms that affect aberrant angiogenesis, expression of genes for VEGF and IGF-1 have an impact on the risk of ROP. In our study, more male infants had sROP than female infants ( $58.8 \%$ vs. $41.2 \%$ ), which is consistent with the findings of Holmström et $\mathrm{al}^{(31)}$ and Darlow et al, ${ }^{(32)}$ but this was not statistically significant in our study $(p=0.372)$. As we did not have genetic data of the subjects, we were unable to retrospectively study this aspect.

Analysis of ophthalmological outcomes was challenging due to the high loss-to-follow-up rates. Many subjects in both groups had defaulted their ophthalmological appointments, with up to $40 \%$ defaulting by three years of age. The reduced sample size for the data at three and four years of age is one of the limitations of this study. Nonetheless, analysis of our data showed that ophthalmological outcomes were similar to those demonstrated in international studies, ${ }^{(33,34)}$ in that structural outcomes following laser treatment for ROP were good. These studies, ${ }^{(35,36)}$ as well as a previous local study, ${ }^{(37)}$ also consistently showed that children with sROP have a high incidence of myopia. While our study demonstrated that the group with sROP had higher percentages of myopia at 1-2 years of age as compared to premature controls, this was not statistically significant. These studies also showed that children with sROP are at increased risk of strabismus, and this was similarly demonstrated in our study, where $50 \%$ of the cases had strabismus (tropias) at four years of age.

Comparing the refractive errors in our population with those in the general population of Singaporean children in the STARS study, ${ }^{(14)}$ our cases with sROP had almost double the 
prevalence of myopia compared to the general population, with $28.6 \%-41.7 \%$ of our cases having myopia at $12-27$ months (two years and three months). In the STARS population, myopia was present in $15.8 \%$ (6-11 months), $14.9 \%$ (12-23 months) and $20.2 \%$ (24-35 months), but the prevalence of myopia dropped considerably at age 36 months and above ( $8.6 \%$ for $36-47$ months and $7.6 \%$ for $48-59$ months). It has been argued that refractive error in children aged below 24 months is both difficult to measure and often overestimated, in part due to incomplete cycloplegia. This drop in myopia prevalence, however, was not seen in our population, as $25.0 \%$ of the cases were still myopic at three and four years old. The prevalence of myopia in preterm controls was also increased at age $3-4$ years $(20.0 \%-21.4 \%)$, suggesting that the incidence of myopia in premature infants was higher than in full-term children, with or without sROP.

In addition, the prevalence rates of astigmatism, anisometropia and strabismus were higher in our study population as compared to the STARS study. ${ }^{(14)}$ The prevalence of astigmatism in our population was significant with $25.0 \%-40.0 \%$ of cases (excluding the first $12-18$ months) and $9.5 \%-40.0 \%$ of controls, as compared to $8.6 \%$ in the STARS study. The prevalence of anisometropia was very low in the STARS study, at only $0.6 \%$, whereas in our population, the prevalence in cases was $7.1 \%-16.7 \%$ (except for four years old) and $7.1 \%$ in the preterm controls at four years old. This suggests that ex-VLBW infants, especially those with laser treatment for ROP, tend to have higher myopia, astigmatism and anisometropia which may progress to amblyopia. This is consistent with the results of the existing literature, which found that incidence of ocular morbidity is higher in ex-premature infants than in full-term infants and is even higher if there is treated ROP. ${ }^{(38)}$

The present study has its limitations. As mentioned, this is a retrospective study and, hence, causal relation is difficult to determine. The higher oxygen requirements and number of blood transfusions may only be a reflection of the other comorbidities such as sepsis or chronic lung disease. Furthermore, this is a small-scale study given the relatively small number of neonates admitted to our hospital, and the lack of data due to subjects defaulting follow-up appointments further limited the study. More research and possible collaboration with other local or Southeast Asian centres to conduct multicentre studies would be helpful to confirm or disprove the findings from this study.

In summary, our study found that an increased number of blood transfusions and higher $\mathrm{FiO}_{2}$ at weeks 7-10 of life (PMA of 33-36 weeks) are significantly associated with sROP in VLBW infants. Therefore, infants who have these risk factors should be vigilantly monitored during the neonatal period to detect any development of retinopathy and given early treatment. The use of oxygen, blood testing and transfusion for premature infants should also be judicious, even up to the tenth week of life. We also found good ophthalmological outcomes, in terms of structure, blindness and refractive errors, for treated ROP. This would help clinicians in reassuring parents of infants during counselling and when explaining outcomes of laser treatment for sROP. However, the importance of follow-up should be emphasised, as children with treated ROP are still at increased theoretical risks of high refractive errors, strabismus and amblyopia. Regular refractive screening after laser treatment is recommended, ${ }^{(39)}$ as such ophthalmological conditions can be treated successfully if detected early.

\section{ACKNOWLEDGEMENTS}

We thank Dr Dimple Rajgor for her assistance in editing, formatting, reviewing and submitting the manuscript for publication.

\section{REFERENCES}

1. Good WV; Early Treatment for Retinopathy of Prematurity Cooperative Group. Final results of the Early Treatment for Retinopathy of Prematurity (ETROP) randomized trial. Trans Am Ophthalmol Soc 2003; 102:233-50.

2. Cryotherapy for Retinopathy of Prematurity Cooperative Group. Multicenter Trial of Cryotherapy for Retinopathy of Prematurity: ophthalmological outcomes at 10 years. Arch Ophthalmol 2001; 119:1110-8.

3. Smith LE. Pathogenesis of retinopathy of prematurity. Growth Horm IGF Res 2004; 14 Suppl A:S140-4.

4. Akkoyun I, Oto S, Yilmaz G, et al. Risk factors in the development of mild and severe retinopathy of prematurity. J AAPOS 2006; 10:449-53.

5. Mccolm JR, Fleck BW. Retinopathy of prematurity: causation. Semin Neonatol 2001; 453-60.

6. Tin W, Gupta S. Optimum oxygen therapy in preterm babies. Arch Dis Child Fetal Neonatal Ed 2007; 92:F143-7.

7. Supplemental Therapeutic Oxygen for Prethreshold Retinopathy of Prematurity (STOP-ROP), a randomized, controlled trial. I: primary outcomes. Pediatrics 2000; 105:295-310.

8. Askie LM, Henderson-Smart DJ, Irwig L, Simpson JM. Oxygen-saturation targets and outcomes in extremely preterm infants. N Engl J Med 2003; 349:959-67.

9. Chen M, Çitil A, McCabe F, et al. Infection, oxygen, and immaturity: interacting risk factors for retinopathy of prematurity. Neonatology 2011; 99:125-32.

10. Hwang JH, Lee EH, Kim EA. Retinopathy of prematurity among very-low-birthweight infants in Korea: incidence, treatment, and risk factors. J Korean Med Sci 2015; 30 Suppl 1:S88-94.

11. Thomas K, Shah P, Canning R, et al. Retinopathy of prematurity: risk factors and variability in Canadian neonatal intensive care units. J Neonatal Perinatal Med 2015; 8:207-14.

12. Wilkinson AR, Haines L, Head K, Fielder AR. UK retinopathy of prematurity guideline. Eye (Lond) 2009; 23:2137-9.

13. The International Committee for the Classification of Retinopathy of Prematurity. The International Classification of Retinopathy of Prematurity revisited. Arch Ophthalmol 2005; 123:991-9.

14. Dirani M, Chan YH, Gazzard G, et al. Prevalence of refractive error in Singaporean Chinese children: the strabismus, amblyopia, and refractive error in young Singaporean Children (STARS) study. Invest Ophthalmol Vis Sci 2010; 51:1348-55

15. Yau GS, Lee JW, Tam VT, et al. Incidence and risk factors of retinopathy of prematurity from 2 neonatal intensive care units in a Hong Kong Chinese population. Asia Pac J Ophthalmol (Phila) 2016; 5:185-91.

16. Lee J, Dammann O. Perinatal infection, inflammation, and retinopathy of prematurity. Semin Fetal Neonatal Med 2012; 17:26-9.

17. Tolsma KW, Allred EN, Chen ML, et al. Neonatal bacteremia and retinopathy of prematurity: the ELGAN study. Arch Ophthalmol 2011; 129:1555-63.

18. Haroon Parupia MF, Dhanireddy R. Association of postnatal dexamethasone use and fungal sepsis in the development of severe retinopathy of prematurity and progression to laser therapy in extremely low-birth-weight infants. J Perinatol $2001 ; 21: 242-7$.

19. Mittal M, Dhanireddy R, Higgins RD. Candida sepsis and association with retinopathy of prematurity. Pediatrics 1998; 101(4 Pt 1):654-7.

20. Dani C, Reali MF, Bertini G, et al. The role of blood transfusions and iron intake on retinopathy of prematurity. Early Hum Dev 2001; 62:57-63.

21. Akter S, Hossain MM, Shirin M, Khalil I, Anwar KS. Blood transfusion: a risk factor in retinopathy of prematurity. Bangladesh J Child Health 2010; 34:38-43.

22. James L, Greenough A, Naik S. The effect of blood transfusion on oxygenation in premature ventilated neonates. Eur J Pediatr 1997; 156:139-41.

23. Cooke RW, Clark D, Hickey-Dwyer M, Weindling AM. The apparent role of blood transfusions in the development of retinopathy of prematurity. Eur J Pediatr 1993; 152:833-6.

24. Hellström A, Smith LE, Dammann O. Retinopathy of prematurity. Lancet 2013; 382:1445-57.

25. Chen ML, Guo L, Smith LE, Dammann CE, Dammann O. High or low oxygen saturation and severe retinopathy of prematurity: a meta-analysis. Pediatrics 
2010; 125:e1483-92.

26. Askie LM, Darlow BA, Schmidt B, et al. Association between oxygen saturation targeting and death or disability in extremely preterm infants in the neonatal oxygenation prospective meta-analysis collaboration. JAMA 2018; 319:2190-201.

27. Wu C, Löfqvist C, Smith LE, VanderVeen DK, Hellström A; WINROP Consortium. Importance of early postnatal weight gain for normal retinal angiogenesis in very preterm infants: a multicenter study analyzing weight velocity deviations for the prediction of retinopathy of prematurity. Arch Ophthalmol 2012; 130:992-9.

28. Binenbaum G, Ying GS, Quinn GE, et al. The CHOP postnatal weight gain birth weight, and gestational age retinopathy of prematurity risk model. Arch Ophthalmol 2012; 130:1560-5.

29. Eckert GU, Fortes Filho JB, Maia M, Procianoy RS. A predictive score for retinopathy of prematurity in very low birth weight preterm infants. Eye (Lond) 2012; 26:400-6.

30. Binenbaum G. Algorithms for the prediction of retinopathy of prematurity based on postnatal weight gain. Clin Perinatol 2013; 40:261-70.

31. Holmström G, van Wijngaarden P, Coster DJ, Williams KA. Genetic susceptibility to retinopathy of prematurity: the evidence from clinical and experimental animal studies. Br J Ophthalmol 2007; 91:1704-8.

32. Darlow BA, Hutchinson JL, Henderson-Smart DJ, et al; Australian and New Zealand Neonatal Network. Prenatal risk factors for severe retinopathy of prematurity among very preterm infants of the Australian and New Zealand Neonatal Network. Pediatrics 2005; 115:990-6.

33. McLoone E, O'Keefe M, McLoone S, Lanigan B. Long term functional and structural outcomes of laser therapy for retinopathy of prematurity. Br J Ophthalmol 2006; 90:754-9.

34. Shah PK, Ramakrishnan M, Sadat B, et al. Long term refractive and structural outcome following laser treatment for zone 1 aggressive posterior retinopathy of prematurity. Oman J Ophthalmol 2014; 7:116-9.

35. O'Connor AR, Stephenson T, Johnson A, et al. Long-term ophthalmic outcome of low birth weight children with and without retinopathy of prematurity. Pediatrics 2002; 109:12-8.

36. Holmström G, El Azazi M, Kugelberg U. Ophthalmological follow up of preterm infants: a population based, prospective study of visual acuity and strabismus. Br J Ophthalmol 1999; 83:143-50.

37. Theng JT, Wong TY, Ling Y. Refractive errors and strabismus in premature Asian infants with and without retinopathy of prematurity. Singapore Med J 2000; 41:393-7.

38. O'Connor AR. Preterm birth: the ophthalmic consequences. Br Ir Orthopt J $2011 ; 8: 3-9$.

39. Ruan L, Shan HD, Liu XZ, Huang X. Refractive status of Chinese with lasertreated retinopathy of prematurity. Optom Vis Sci 2015; 92(4 Suppl 1):S3-9. 